\title{
Long non-coding RNA polymorphisms on 8 q24 are associated with the prognosis of gastric cancer in a Chinese population
}

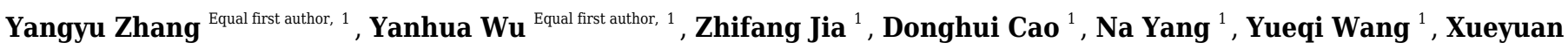 \\ Cao ${ }^{\text {Corresp., }}{ }^{2}$, Jing Jiang ${ }^{\text {Corresp. } 1}$ \\ 1 Division of Clinical Research, First Hospital of Jilin University, Changchun, Jilin, China \\ 2 Department of Gastric and Colorectal Surgery, University Hospital of South Manchester, Changchun, Jilin, China \\ Corresponding Authors: Xueyuan Cao, Jing Jiang \\ Email address: caoxy@aliyun.com, jiangjing19702000@jlu.edu.cn
}

Background. Gastric cancer (GC) remains the third leading cause of cancer death in China. Although genome-wide association studies (GWASs) have identified the association between several single nucleotide polymorphisms (SNPs) on 8q24 and the risk of GC, the role of these SNPs in the prognosis of GC in Chinese populations has not yet been fully evaluated. Therefore, this study was conducted to explore the association between long non-coding RNA (InCRNA) polymorphisms on 8q24 and the prognosis of GC.

Methods. We genotyped 726 surgically resected GC patients to explore the association between eight SNPs in the IncRNAs CCAT1 (rs10087719, rs7816475), PCAT1 (rs1026411), PRNCR1 (rs12682421, rs13252298), and CASC8 (rs1562430, rs4871789, rs6983267) transcribed from the 8q24 locus and the prognosis of GC in a Chinese population.

Results. We found that the patients carrying rs12682421 AA genotypes survived for a shorter time than those with the GG/GA genotype (HR=1.39, 95\% Cl: 1.09-1.78). Compared with the CC/CT genotype, the $\Pi$ genotype of rs 1562430 was associated with an increased risk of death ( $\mathrm{HR}=1.38,95 \% \mathrm{Cl}: 1.06-1.80)$. Furthermore, the results also identified the rs1026411 SNP as an independent prognostic factor for poor survival in GC patients. Patients carrying AA/AG variant genotypes had a $36 \%$ increased risk of death compared to those carrying the $\mathrm{GG}$ genotype ( $\mathrm{HR}=1.36,95 \% \mathrm{Cl}$ : 1.06-1.74). These findings suggested that the rs12682421, rs1026411 and rs1562430 SNPs may contribute to the survival of GC and be prognostic markers for GC. 
1 Long non-coding RNA polymorphisms on 8q24 are

2 associated with the prognosis of gastric cancer in a

3 Chinese population

4 Yangyu Zhang ${ }^{1 *}$, Yanhua $\mathrm{Wu}^{1 *}$, Zhifang $\mathrm{Jia}^{1}$, Donghui $\mathrm{Cao}^{1}$, Na Yang ${ }^{1}$, Yueqi Wang ${ }^{1}$, Xueyuan

$5 \mathrm{CaO}^{2 \#}$, and Jing Jiang ${ }^{1 \#}$

6

$7{ }^{1}$ Division of Clinical Research, First Hospital of Jilin University, Changchun, Jilin Province,

8 China

92 Department of Gastric and Colorectal Surgery, First Hospital of Jilin University, Changchun,

10 Jilin, China

11

12

${ }^{*}$ Contributed equally.

13

Corresponding Author:

15 Xueyuan $\mathrm{CaO}^{2}$

16

71 Xinmin Street, Changchun, Jilin Province, 130021, China.

Email address: caoxy@aliyun.com;

18 Jing Jiang ${ }^{1}$

71 Xinmin Street, Changchun, Jilin Province, 130021, China.

Email address: jiangjing19702000@jlu.edu.cn. 
37

38

39

40

41

42

43

44

45

46

47

48

49

50

51

52

53

54

55

60

61

62

63

64

65

66

67

68

\section{Abstract}

Background. Gastric cancer (GC) remains the third leading cause of cancer death in China. Although genome-wide association studies (GWASs) have identified the association between several single nucleotide polymorphisms (SNPs) on 8q24 and the risk of GC, the role of these

SNPs in the prognosis of GC in Chinese populations has not yet been fully evaluated. Therefore, this study was conducted to explore the association between long non-coding RNA (lncRNA) polymorphisms on $8 \mathrm{q} 24$ and the prognosis of GC.

Methods. We genotyped 726 surgically resected GC patients to explore the association between eight SNPs in the 1ncRNAs CCAT1 (rs10087719, rs7816475), PCAT1 (rs1026411), PRNCR1 (rs12682421, rs13252298), and CASC8 (rs1562430, rs4871789, rs6983267) transcribed from the $8 \mathrm{q} 24$ locus and the prognosis of GC in a Chinese population.

Results. We found that the patients carrying rs12682421 AA genotypes survived for a shorter time than those with the GG/GA genotype ( $\mathrm{HR}=1.39,95 \% \mathrm{CI}: 1.09-1.78)$. Compared with the $\mathrm{CC} / \mathrm{CT}$ genotype, the TT genotype of rs1562430 was associated with an increased risk of death $(\mathrm{HR}=1.38,95 \% \mathrm{CI}: 1.06-1.80)$. Furthermore, the results also identified the rs $1026411 \mathrm{SNP}$ as an independent prognostic factor for poor survival in GC patients. Patients carrying AA/AG variant genotypes had a $36 \%$ increased risk of death compared to those carrying the GG genotype (HR=1.36, 95\% CI: 1.06-1.74). These findings suggested that the rs12682421, rs1026411 and rs1562430 SNPs may contribute to the survival of GC and be prognostic markers for GC. 


\section{Introduction}

Gastric cancer (GC) is the fifth most common malignancy and the third leading cause of cancerrelated mortality in the world. A total of $1,033,701$ new cases of GC were estimated to have occurred in 2018 (Bray et al. 2018). Although the incidence of GC has been declining in the last decades in most regions, it remains a common cancer among many populations in East Asia. Due to a high incidence rate and a large population, more than $40 \%$ of GC cases worldwide have occurred in China, according to GLOBOCAN 2012 (Ferlay et al. 2015). In the last decade, the mortality rate of GC has declined conspicuously due to the improved treatment approaches, but the prognosis of GC is still poor; the five-year survival rate is $29.0 \%$ (Zeichner et al. 2017).

Regarding treatment approaches, tumorectomy with adjuvant or neoadjuvant chemotherapy and radiotherapy are the most effective treatments for GC. However, despite improvements in surgical and adjuvant multimodal treatments, the prognosis of GC is still poor due to late diagnosis and extreme intra- and inter-tumour heterogeneity (Bonelli et al. 2019). The heterogeneity makes the selection of treatment options difficult, and previous studies have found that patients with the same pathological stage and tumour grade who receive similar therapies may have different clinical outcomes, a finding that indicates the significance of individual variants influenced by genetic and environmental factors (Wang et al. 2018). Therefore, exploring genetic variations in key genes involved in tumour progression as biomarkers to improve the prognosis prediction of GC patients is imperative.

The results from genome-wide association studies (GWASs) have also identified single nucleotide polymorphisms (SNPs), the most common type of genetic variations in the human genome, in relation to the tumourigenesis of GC (Abnet et al. 2010; Sakamoto et al. 2008; Shi et al. 2011). GWASs have identified several loci, including 1q22, 5p13.1 and 8q24, that are associated with GC susceptibility, mainly in populations in Asia (Saeki et al. 2013; Shi et al. 2011; Wadhwa et al. 2013). Particularly, a series of evidence has suggested that 8q24 chromosome region can not only affect GC susceptibility (Zhi et al. 2017), but also confer GC patients with different prognosis (Ma et al. 2015b; Wang et al. 2016), which further verified that genetic background play an important role in gastric carcinogenesis and progression.

Although paying attention to known genes might generate further understanding in development and therapy of GC, newly-developed markers such as long non-coding RNAs (lncRNAs) may lead novel insight into the mechanism of GC risk or treatment. LncRNAs are noncoding transcripts that are more than 200 nucleotides long. Although they were initially regarded as "transcriptional noise", increasing studies have found that IncRNAs can regulate local or global gene expression through transcriptional, post-transcriptional and epigenetic 
105

106

107

108

109

110

111

112

113

114

115

116

117

118

119

120

121

122

123

124

125

126

127

128

129

130

131

132

133

134

135

136

137

138

139

140

regulation (Mercer et al. 2009). As lncRNAs play multiple roles in the regulation of gene expression, aberrant lncRNA expression may therefore occur during carcinogenesis and disease development. These advantages make lncRNAs potential biomarkers for the diagnosis, prognosis and therapy of a variety of cancers, including GC (Wu \& Hsieh 2019; Yuan et al. 2018; Zhao et al. 2015c).

The $8 \mathrm{q} 24$ chromosome region has been reported to express several lncRNAs in different human tumours (Huang et al. 2018; Tong et al. 2018; Xiang et al. 2014). The association between polymorphisms in lncRNAs and the risk of gastric cancer has been studied in several ethnicities (Labrador et al. 2015; Pan et al. 2016; Y et al. 2017). However, there are few studies that have investigated the prognostic value of lncRNA polymorphisms on 8q24 in GC patients. Hence, this study was performed to examine whether variants of lncRNA colon cancerassociated transcript (CCAT1), prostate cancer-associated transcript 1 (PCAT1), prostate cancer non-coding RNA 1 (PRNCR1) and cancer susceptibility candidate 8 (CASC8) genes on chromosome $8 \mathrm{q} 24$ are associated with survival in a Chinese population with GC. It may bring benefits for individualized treatment and consequently improve survival outcomes.

\section{Materials \& Methods}

\section{Study population}

Subjects of the study were the newly diagnosed gastric cancer patients recruited from the Department of Gastric and Colorectal Surgery of the First Hospital of Jilin University from 2008 to 2013. A total of 756 patients who underwent tumourectomy without receiving chemotherapy or radiotherapy before surgery were enrolled in this study. The individual characteristics (gender, age) and clinical data (tumour size, histological type, histological grade, lymph metastasis, distant metastasis, depth of invasion, neural invasion and therapy) were collected from the medical records. TNM classification, based on the 2010 seventh edition of the American Joint Committee on Cancer (AJCC) guidelines(Washington 2010), was used to evaluate the clinical stage of the cancer. The evaluation of $H$. pylori infection was performed via a serum immunoglobulin $\mathrm{G}$ (IgG) antibody test by an enzyme-linked immunosorbent assay (ELISA) using an H. pylori-IgG ELISA kit (Biohit, Helsinki, Finland). Postoperative chemotherapy was identified as an effective therapy for at least 3 cycles.

\section{Ethics Statement}

Each patient in this study signed an informed consent form before sample and information collection. This study was approved by the ethics committees of the First Hospital of Jilin University.

\section{Follow-up}

The follow-up of the patients was conducted 3 months, 6 months, and 1 year after surgery and every 1 year thereafter until the death of the patient or loss to follow-up. The data from each 
follow-up visit were collected. Subjects were excluded if they were lost to follow-up at the first phone interview or died due to complications of the surgery during the perioperative period (within 30 days after surgery). The survival time was considered as the duration (i) from the date of the surgery to the date of death if the GC patient had died or (ii) from the date of the surgery to the date of the last phone interview if the patient was lost to follow-up or to the end of the study if the patient was still alive.

\section{Tagging SNP selection}

From whole blood sample of each patient, we extracted genomic DNA using a MagPure Tissue and Blood DNA KF Kit (Magen, Guangzhou, China). The tag SNPs and the well-studied SNPs on 8q24 that were previously reported be associated with gastrointestinal tumours were selected. These SNPs included CCAT1 rs10087719, CCAT1 rs7816475, PCAT1 rs1026411, PRNCR1 rs12682421, PRNCR1 rs13252298, CASC8 rs1562430, CASC8 rs4871789 and CASC8 rs6983267. The SNPinfo (http://snpinfo.niehs.nih.gov/), GVS (http://gvs.gs.washington.edu/GVS147/) and F-SNP (http://compbio.cs.queensu.ca/F-SNP/) databases were used to select tag SNPs. The minor allele frequency (MAF) of all the SNPs was > 0.05 based on the Han Chinese Population.

\section{Genotyping}

SNP genotyping was conducted by the MassARRAY technology platform (Sequenom, CA, USA) and was determined by the Bio Miao Biological Technology Co., Ltd. (Beijing). The detection rates for rs10087719, rs7816475, rs1026411, rs12682421, rs13252298, rs1562430, rs4871789 and rs 6983267 were $100 \%, 98 \%, 99 \%, 100 \%, 100 \%, 100 \%, 98 \%$, and $94 \%$, respectively. The linkage disequilibrium (LD) was established with a threshold of the pairwise $r^{2}$ coefficient greater than 0.80 and the extent of LD between the eight SNPs were estimated using Haploview 4.2 (Broad Institute of MIT and Harvard, Cambridge, MA, USA). None of the eight SNPs were located at CCAT1, PRNCR1 or CASC8 with LD.

\section{Transcription factor binding site prediction}

Transcription factor binding sites (TFBS) were predicted using the PROMO database (http://alggen.lsi.upc.es/cgi-bin/promo_v3/promo/promoinit.cgi?dirDB=TF_8.3).

\section{Statistical Analysis}

Frequency and proportion were used to describe the categorical variables. A goodness of fit $\chi^{2}$ test was used to test the Hardy-Weinberg equilibrium (HWE) of each SNP. Survival curves of the GC patients based on each SNP were plotted by the Kaplan-Meier method and were compared by log-rank test. The Cox regression model was used to calculate hazard ratios (HRs) with $95 \%$ confidence intervals (CIs) and to evaluate the associations between genotypes of each SNP and overall survival after adjusting for potential confounders (age, gender, H. pylori, 
tumour size, TNM stage, histological type, histological grade, chemotherapy, lymph vascular invasion and neural invasion). We used the Bonferroni correction method to adjust for multiple testing with a significance threshold set at $P=0.00625(0.05 / 8$ SNPs). All statistical analyses were performed using the SPSS 21.0 software (IBM SPSS, IBM Corp, Armonk, NY, USA).

\section{Results}

\section{Characteristics of patients}

A total of 756 diagnosed GC patients were enrolled in the study. Fourteen patients died of complications from the surgery during the preoperative period, seven patients were lost to follow-up at the first phone interview and the genotyping of nine patients failed. The remaining 726 patients were included in the study for the subsequent analysis. At the end of the study, 27 patients were lost to follow-up, 357 patients died, and 342 patients were alive (Fig. 1). The duration of follow-up was from 1 month to 109 months, and the median follow-up time was 70.7 months. The characteristics of the 726 patients are shown in Table 1.

\section{Genotype and allele frequencies of the eights SNPs}

The genotype frequencies of seven SNPs (rs10087719, rs7816475, rs1026411, rs12682421, rs 13252298, rs1562430, rs4871789) in the subjects were in HWE with non-significant $\chi^{2}$ values $(P>0.05)$. The rs6983267 locus, however, was found to deviate from the HWE $(P<0.001)$. We randomly selected $10 \%$ of the samples for repeat genotyping of rs6983267, but the result remained the same. The distributions of the genotype and allele frequencies of the eight SNPs in subjects are shown in Table 2. After Bonferroni correction for multiple testing, none of the eight SNPs were significantly associated with the survival of GC. However, there was a statistically significant tendency for rs12682421 (log-rank $P=0.03$ ). As shown in Table 2, patients with the rs 12682421 GA genotype tended to have a better prognosis $(\mathrm{HR}=0.77,95 \% \mathrm{CI}$ : $0.61-0.97)$ than patients with the AA genotype.

\section{Multivariate Cox regression analysis of SNPs and gastric cancer survival}

A multivariate stepwise Cox regression model was performed to explore the independent prognostic factor for GC. The results showed that tumour size, TNM stage, lymph vascular invasion and chemotherapy were associated with the survival of the patients. The AA genotypes of rs 12682421 were associated with a significantly increased risk of death compared with that of the GG/GA genotype $(\mathrm{HR}=1.39,95 \% \mathrm{CI}$ : 1.09-1.78). The TT genotypes of rs 1562430 were also associated with increased risk of death compared with that of the CC/CT genotype $(\mathrm{HR}=1.38,95 \%$ CI: 1.06-1.80). The results also identified rs1026411 SNP as an independent prognostic factor for the poor survival of GC patients; patients carrying AA/AG genotypes had a 36\% increased risk of death compared to those carrying the GG genotype $(\mathrm{HR}=1.36,95 \% \mathrm{CI}$ : 1.06-1.74) (Table 3). The survival curves of the three SNPs are shown in Fig. 2.

\section{Stratified analysis of the genotypes associated with gastric cancer prognosis}


212 Moreover, the associations between the three SNPs (rs12682421, rs1562430, rs1026411) and the

213 survival of the GC patients was evaluated by a stratified analysis of tumour size, TNM stage,

214 lymph vascular invasion and chemotherapy. Compared to patients with the GA/GG genotype of

215 rs12682421, patients with the AA variant genotype had a higher death risk in the subgroup of

216 patients with tumour sizes $<5 \mathrm{~cm}(\mathrm{HR}=1.59,95 \% \mathrm{CI}: 1.11-2.28)$, an advanced TNM stage (TNM

217 stage II: $\mathrm{HR}=1.68,95 \% \mathrm{CI}: 1.03-2.76$; TNM stage III: HR=1.41, 95\%CI: 1.05-1.90), lymph

218 vascular invasion $(\mathrm{HR}=1.49,95 \% \mathrm{CI}: 1.15-1.95)$ or no postoperative chemotherapy $(\mathrm{HR}=1.40$,

219 95\%CI: 1.01-1.94) (Fig. 3A). Compared to patients with the CC/CT genotype, patients carrying

220 TT genotypes of rs 1562430 had a higher death risk in the subgroup of patients with tumour sizes

$221 \geq 5 \mathrm{~cm}(\mathrm{HR}=1.65,95 \% \mathrm{CI}: 1.14-2.38)$, TNM stage III (HR=1.57, 95\%CI: 1.12-2.20), or lymph

222 vascular invasion ( $\mathrm{HR}=1.55,95 \% \mathrm{CI}: 1.16-2.07)$ and in the subgroup of patients who did not

223 receive postoperative chemotherapy (HR=1.48, 95\%CI: 1.04-2.10) (Fig. 3B). Compared to

224 patient with the GG genotype of rs1026411, patients with the AA/AG variant genotype had a

225 higher death risk in the subgroup of patients with tumour sizes $\geq 5 \mathrm{~cm}$ (HR=1.55, 95\%CI: 1.09 -

226 2.21) or lymph vascular invasion ( $\mathrm{HR}=1.43$, 95\%CI: 1.09-1.87) (Fig. 3C).

\section{Discussion}

228 Clinically, therapeutic decision making and prognostic prediction for GC patients still depend on the TNM staging system. However, due to the significant heterogeneity within the same stage, the TNM stage alone is not sufficient to predict the prognosis of GC. More significantly, although the TNM staging system classifies patients into subgroups with different clinical outcomes, it provides limited information about therapeutic effects in individual patients (Li et al. 2017; Qu et al. 2015). Therefore, it is crucial and necessary to identify new biomarkers for GC patients to complement the TNM staging system in order to improve the prediction of prognosis and guide therapeutic decisions. Our study focused on the investigation of eight lncRNA SNPs on $8 \mathrm{q} 24$ that predispose GC patients to survival. Multivariate analysis revealed that the AA genotype of rs12682421, the TT genotype of rs1562430 and the AA/AG genotype of rs1026411 could serve as potential markers to predict the unfavourable survival of GC patients in the Chinese population. Furthermore, the prominent prognostic effect of the three SNPs was more evident in advanced subgroups of GC patients.

Recent research has suggested that lncRNAs, as oncogenes or tumour suppressor genes, could be involved in the development of cancer and be associated with tumour metastasis and prognosis (Song et al. 2017; Weidle et al. 2017). Given that the majority of the GWAS-identified cancer risk SNPs are located in the noncoding region, the expression and function of lncRNAs are more likely to be impacted by the SNPs (Gao \& Wei 2017). Moreover, GWAS have identified 8q24 as a hotspot for cancer-associated SNPs on account of the strength, density and high allele frequency of these SNPs (Sur et al. 2013). Although several studies have revealed that 1ncRNAs on 8q24, including PRNCR1, CCAT2 and PCAT1, encompass the cancer predisposition SNPs (Guo et al. 2019; Ling et al. 2013; Wang \& Wang 2019; Yang et al. 2019), the prognostic significance of these lncRNAs in GC patients has not yet been fully explored. 
252

253

254

255

256

257

258

259

260

261

262

263

264

265

266

267

268

269

270

271

272

273

274

275

276

277

278

279

280

281

282

283

284

285

286

287

288

289

290

291

cancer (PCa) by activating androgen receptor (AR) (Chung et al. 2011); in addition, polymorphisms of the lncRNA PRNCR1 were noted in many cancers, including colorectal cancer (AlMutairi \& Parine 2019), prostate cancer (Sattarifard et al. 2017), and gastric cancer (He et al. 2017). A meta-analysis conducted by Huang et al. (Huang et al. 2018) showed that rs 16901946 of PRNCR1, which was in complete LD with rs12682421, was associated with an increased risk of gastric cancer in the dominant model. A study performed by He et al. (He et al. 2017) that aimed to assess the GC susceptibility and GC prognostic value of the polymorphisms in PRNCR1, found that rs16901946 G allele carriers (linked with rs12682421 G allele) have an increased risk of $\mathrm{GC}$, but this polymorphism did not exhibit any significant prognostic value for GC. However, in our study, we found that compared with the GA/GG genotype, the PRNCR1 rs 12682421 AA genotype was a poor prognostic factor for $\mathrm{GC}$, which is different from the results of the study by $\mathrm{He}$ et al. We considered that the reasons for the inconsistent conclusion may be due to the fact that doctor He's study has a smaller sample size $(\mathrm{N}=494)$, a shorter follow-up time (the patients were followed for up to 4 years) and fewer events compared with our study.

Rs1562430 is located in the intron of CASC8, a long noncoding RNA (lncRNA), and overlaps with the POU5F1B gene. Previous studies have revealed that the rs 1562430 SNP has a strong association with the risk of breast cancer and colorectal cancer (He et al. 2011; Kim et al. 2012; Silvestri et al. 2015). Ma et al. (Ma et al. 2015a) found that there were no associations between the rs1562430 genotype and the survival of GC patients in a Chinese population. However, in the present study, we found that rs1562430 TT was associated with a significantly lower survival rate in $\mathrm{GC}$ patients than $\mathrm{CC} / \mathrm{CT}$. The study conducted by Ma et al. included patients with TNM stage IV, and the histological type of $42.5 \%$ of patients was intestinal. Conversely, our study excluded patients with TNM stage IV, and more than $70 \%$ of patients exhibited an intestinal histological type. Moreover, nearly $70 \%$ of the patients in our study were infected with $H$. pylori. Therefore, the differences in the pathogenic environment and genetic background of the patients included in the two studies may be the reasons for the inconsistent results.

Existing studies have found that PCAT1 overexpression occurred in PCa, lung cancer and colorectal cancer (Ge et al. 2013; Prensner et al. 2011; Zhao et al. 2015a). Shi et al. (Shi et al. 2015) identified that ESCC patients with high levels of PCAT1 had poorer survival times than those with low levels of PCAT1. Moreover, recent studies have suggested that a PCAT1 genetic variant may play an essential role in the susceptibility to several cancers (Ren et al. 2017; Zhao et al. 2015b). Yuan et al. (Yuan et al. 2018) found that rs1902432 in PCAT1 was significantly associated with an increased risk of PCa, and Lin et al. (Lin et al. 2017) found that rs710886 of PCAT1 was significantly associated with bladder cancer risk in a Chinese population. As far as we know, no study has been conducted on the role of PCAT1 polymorphisms in the prognosis of GC. In the present study, we found that, compared to GG, rs1026411 AA/AG was associated with a poor prognosis of GC patients (HR: 1.33, 95\% CI: 1.03-1.70).

Previous studies have found that SNPs in lncRNAs have different prognostic values when they occur with different clinical features (Ma et al. 2015a; Xiong et al. 2017); therefore, we

Peer) reviewing PDF | (2019:09:41373:1:0:REVIEW 3 Jan 2020) 
292

293

294

295

296

297

298

299

300

301

302

303

304

305

306

307

308

309

310

311

312

313

314

315

316

317

318

319

320

321

322

323

324

325

326

327

328

329

330

331

332

conducted a stratified analysis by tumour size, TNM stage, lymph vascular invasion and chemotherapy. The unfavourable prognostic effects of rs1026411, rs1562430 and rs12682421 were more evident among patients with increased TNM stage and lymph vascular invasion, which indicated that these three SNPs may have higher predictive value in advanced stages of GC. Previous studies have demonstrated that genomic polymorphisms can affect drug transport, metabolism and cellular response and cause individual variations in terms of the response and even overall survival (Ulrich et al. 2003). Increasing evidence has also suggested that SNPs in some lncRNAs are related to chemotherapy response and could provide effective therapeutic targets for GC treatment (Ozgur et al. 2019; Zhang \& Du 2016). Our results showed that in patients who did not receive chemotherapy, the rs12682421 AA genotype and rs1562430 TT genotype could predict poor survival ( $\mathrm{HR}=1.40,95 \% \mathrm{CI}: 1.01-1.94$; $\mathrm{HR}=1.48,95 \% \mathrm{CI}: 1.04-2.10)$; however, in the subgroup of patients who received chemotherapy, the difference in the genomic polymorphisms of the two SNPs disappeared. The effect of the genetic background of the two SNPs may be overshadowed by the advantages of chemotherapy, considering that the multivariate analysis results showed that patients with postoperative chemotherapy tended to have a favourable prognosis (HR: $0.72,95 \%$ CI: $0.56-0.91$ ).

Because lncRNAs could play a crucial role in the regulation of gene expression via transcription and transcription factors often play important roles in tumourigenesis, we used bioinformatics data from the PROMO transcription factor binding site database to predict the possible functions of rs 12682421, rs1562430 and rs1026411. We found that the $\mathrm{C}$ allele at the rs 1562430 locus allowed binding to the glucocorticoid receptor $\alpha(\mathrm{GR} \alpha)$, which is a transcription factor that increases genes that participate in cell cycle arrest and apoptosis (Kumar et al. 2004; Patki \& McFall 2018; Yemelyanov et al. 2006). GR $\alpha$ could be bound and activated by glucocorticoids, and previous studies have shown that a higher expression of glucocorticoids receptors has been correlated with a better prognosis in bladder cancer (Ishiguro et al. 2014; Zheng et al. 2012). Therefore, we considered that the rs $1562430 \mathrm{~T}$ allele may be associated with a lower GR $\alpha$ expression, which affects the prognosis of the GC patients. Additionally, the A allele at the rs 12682421 locus was found to be allowed binding to the GR $\beta$ transcription factor, which is a different isoform of GR. GR $\beta$ lacks the ligand-binding domain for glucocorticoids (Hinds et al. 2010) and has been indicated to inhibit GR $\alpha$ (D Y et al. 1997; Hinds et al. 2010; Kubin et al. 2016). GR $\beta$ has been demonstrated to be involved in the migration of bladder cancer and brain cancer (Mcbeth et al. 2016; Ying et al. 2013), and some other studies have also reported that GR $\beta$ levels are elevated in inflammatory diseases and cancers, leading to increased progression (Jin et al. 2007; Marino et al. 2016; Psarra et al. 2005). Hence, we hypothesize that the poor prognosis of patients with the rs 12682421 A allele may be associated with a higher GR $\beta$ expression. Moreover, we found that the $\mathrm{G}$ allele at the rs 1026411 locus facilitated binding to the polyomavirus enhancer activator 3 (PEA3) transcription factor, which belongs to the PEA3 subfamily within the E-twenty-six (ETS) domain transcription factor superfamily (Kandemir et al. 2017). Members of the PEA3 subfamily have been demonstrated in previous studies be associated with a variety of cancers (Cowden-Dahl \& Zeineldin 2007; Keld et al. 2010; Kim et al. 2015), but a study conducted by Keld et al. (Keld et al. 2011) showed that PEA3

Peer) reviewing PDF | (2019:09:41373:1:0:REVIEW 3 Jan 2020) 
333 upregulation in isolation does not predict prognosis in any stage of gastric cancer. The specific

334

335

336

337

338

339

340

341

342

343

344

345

346

347

348

349

350

351

352

353

354

355

356

357

358

359

360

361

362

363

364

365

366

367

368

369

370

roles of GR $\alpha$, GR $\beta$ and PEA3 in gastric cancer should be verified in further studies.

There are several limitations in present study that should be noted. First, although the median follow-up time was 70.7 months, more than half of the patients survived, and the number of events was insufficient, which may limit the statistical power of our findings. Second, despite the fact that we found associations between three SNPs and overall survival of GC, the mechanisms are still not clear and need to be further elucidated. Third, our study was based on a single group of patients. Hence, other independent replications and multi-centre studies need to be done to explore the role of genetic polymorphisms of lncRNA on 8q24 in the prognosis of GC in different populations.

\section{Conclusions}

In summary, the present study revealed that the PRNCR1 rs12682421 AA genotype, the CASC8 rs1562430 TT genotype and the PCAT1 rs1026411 AA/AG genotype could serve as potential markers to predict the unfavourable survival of GC patients in the Chinese population. These three SNPs may be used as prognostic markers in combination with traditional clinical prognostic factors to refine therapeutic decisions for the individualised treatment of GC.

\section{Acknowledgements}

We thank Ying Song for her work on follow-up of participants and the group of Gastric and Colorectal Surgery for their support.

\section{References}

Abnet CC, Freedman ND, Hu N, Wang Z, Yu K, Shu XO, Yuan JM, Zheng W, Dawsey SM, and Dong LM. 2010. A shared susceptibility locus in PLCE1 at 10q23 for gastric adenocarcinoma and esophageal squamous cell carcinoma. Nature Genetics 42:764.

AlMutairi M, and Parine NR. 2019. Association between polymorphisms in PRNCR1 and risk of colorectal cancer in the Saudi population. 14:e0220931. 10.1371/journal.pone.0220931

Bonelli P, Borrelli A, Tuccillo FM, Silvestro L, Palaia R, and Buonaguro FM. 2019. Precision medicine in gastric cancer. World journal of gastrointestinal oncology 11:804-829. 10.4251/wjgo.v11.i10.804

Bray F, Ferlay J, Soerjomataram I, Siegel RL, Torre LA, and Jemal A. 2018. Global cancer statistics 2018: GLOBOCAN estimates of incidence and mortality worldwide for 36 cancers in 185 countries. CA Cancer J Clin 68:394424. 10.3322/caac. 21492

Chung S, Nakagawa H, Uemura M, Piao L, Ashikawa K, Hosono N, Takata R, Akamatsu S, Kawaguchi T, Morizono T, Tsunoda T, Daigo Y, Matsuda K, Kamatani N, Nakamura Y, and Kubo M. 2011. Association of a novel long non-coding RNA in 8q24 with prostate cancer susceptibility. Cancer Sci 102:245-252. 10.1111/j.13497006.2010.01737.x

Cowden-Dahl K, and Zeineldin R, Lg. 2007. PEA3 is necessary for optimal epidermal growth factor receptor-

Peer) reviewing PDF | (2019:09:41373:1:0:REVIEW 3 Jan 2020) 
stimulated matrix metalloproteinase expression and invasion of ovarian tumor cells. Molecular Cancer Research 5:413-421.

D Y L, Q H, A V, S J S, W S, E M, G P C, and D J K. 1997. Association of glucocorticoid insensitivity with increased expression of glucocorticoid receptor beta. The Journal of Experimental Medicine 186:1567-1574.

Ferlay J, Soerjomataram I, Dikshit R, Eser S, Mathers C, Rebelo M, Parkin DM, Forman D, and Bray F. 2015. Cancer incidence and mortality worldwide: sources, methods and major patterns in GLOBOCAN 2012. Int J Cancer 136:E359-386. 10.1002/ijc.29210

Gao P, and Wei GH. 2017. Genomic Insight into the Role of IncRNAs in Cancer Susceptibility. International Journal of Molecular Sciences 18.

Ge X, Chen Y, Liao X, Liu D, Li F, Ruan H, and Jia W. 2013. Overexpression of long noncoding RNA PCAT-1 is a novel biomarker of poor prognosis in patients with colorectal cancer. Medical Oncology 30:588.

Guo Q, Lv S, Wang B, Li Y, Cha N, Zhao R, Bao W, and Jia B. 2019. Long non-coding RNA PRNCR1 has an oncogenic role in breast cancer. Exp Ther Med 18:4547-4554. 10.3892/etm.2019.8152

He BS, Sun HL, Xu T, Pan YQ, Lin K, Gao TY, Zhang ZY, and Wang SK. 2017. Association of Genetic Polymorphisms in the LncRNAs with Gastric Cancer Risk in a Chinese Population. J Cancer 8:531-536.

He J, Wilkens LR, Stram DO, Kolonel LN, Henderson BE, Wu AH, Le ML, and Haiman CA. 2011. Generalizability and epidemiologic characterization of eleven colorectal cancer GWAS hits in multiple populations. Cancer epidemiology, biomarkers \& prevention : a publication of the American Association for Cancer Research, cosponsored by the American Society of Preventive Oncology 20:70.

Hinds TD, Sadeesh R, Cash HA, Stechschulte LA, Garrett H, Najjar SM, and Sanchez ER. 2010. Discovery of glucocorticoid receptor-beta in mice with a role in metabolism. Molecular Endocrinology 24:1715.

Huang X, Zhang W, and Shao Z. 2018. Association between long non-coding RNA polymorphisms and cancer risk: a meta-analysis. Biosci Rep:BSR20180365.

Ishiguro H, Kawahara T, Netto G, and Miyamoto H. 2014. MP50-15 REDUCED GLUCOCORTICOID RECEPTOR EXPRESSION PREDICTS BLADDER TUMOR RECURRENCE AND PROGRESSION. Journal of Urology 191:e499e499.

Jin Z, Gong JY, Jr OBG, Cartegni L, Nanus DM, and Shen R. 2007. Bombesin attenuates pre-mRNA splicing of glucocorticoid receptor by regulating the expression of serine-arginine protein p30c (SRp30c) in prostate cancer cells. Biochimica Et Biophysica Acta 1773:1087-1094.

Kandemir B, Dag U, Bakir Gungor B, Durasi IM, Erdogan B, Sahin E, Sezerman U, and Aksan Kurnaz I. 2017. In silico analyses and global transcriptional profiling reveal novel putative targets for Pea3 transcription factor related to its function in neurons. Plos One 12:e0170585. 10.1371/journal.pone.0170585

Keld R, ., Guo B, ., Downey P, ., Cummins R, ., Gulmann C, ., Ang YS, and Sharrocks AD. 2011. PEA3/ETV4-related transcription factors coupled with active ERK signalling are associated with poor prognosis in gastric adenocarcinoma. British Journal of Cancer 105:124-130.

Keld R, Guo B, Downey P, Gulmann C, Ang YS, and Sharrocks AD. 2010. The ERK MAP kinase-PEA3/ETV4-MMP-1 axis is operative in oesophageal adenocarcinoma. Molecular Cancer 9:313.

Kim HC, Lee JY, Sung H, Choi JY, Park SK, Lee KM, Kim YJ, Min JG, Li L, and Cho YS. 2012. A genome-wide association study identifies a breast cancer risk variant in ERBB4 at 2q34: results from the Seoul Breast Cancer Study. Breast Cancer Research Bcr 14:R56.

Kim HJ, Kim SH, Yu EJ, Seo WY, and Kim JH. 2015. A positive role of DBC1 in PEA3-mediated progression of estrogen 
receptor-negative breast cancer. Oncogene 34:4500-4508. 10.1038/onc.2014.381

Kubin ME, Hagg PM, Kokkonen N, Vayrynen JP, Haapasaari KM, Moilanen J, Kallioinen M, Hurskainen T, and Tasanen K. 2016. Glucocorticoid receptors GRalpha and GRbeta are expressed in inflammatory dermatoses. Eur J Dermatol 26:21-27. 10.1684/ejd.2015.2691

Kumar R, Johnson BH, and Thompson EB. 2004. Overview of the structural basis for transcription regulation by nuclear hormone receptors. Essays in Biochemistry 40:27-39.

Labrador L, Torres K, Camargo M, Santiago L, Valderrama E, and Chiurillo MA. 2015. Association of common variants on chromosome 8q24 with gastric cancer in Venezuelan patients. Gene 566:120-124. 10.1016/j.gene.2015.04.081

Li Q, Qu F, Li R, He X, Zhai Y, Chen W, and Zheng Y. 2017. A functional polymorphism of SSBP1 gene predicts prognosis and response to chemotherapy in resected gastric cancer patients. Oncotarget 8:110861110876. 10.18632/oncotarget.22864

Lin Y, Ge Y, Wang Y, Ma G, Wang X, Liu H, Wang M, Zhang Z, and Chu H. 2017. The association of rs710886 in IncRNA PCAT1 with bladder cancer risk in a Chinese population. Gene 627.

Ling H, Spizzo R, Atlasi Y, Nicoloso M, Shimizu M, Redis RS, Nishida N, Gafa R, Song J, Guo Z, Ivan C, Barbarotto E, De Vries I, Zhang X, Ferracin M, Churchman M, van Galen JF, Beverloo BH, Shariati M, Haderk F, Estecio MR, Garcia-Manero G, Patijn GA, Gotley DC, Bhardwaj V, Shureiqi I, Sen S, Multani AS, Welsh J, Yamamoto K, Taniguchi I, Song MA, Gallinger S, Casey G, Thibodeau SN, Le Marchand L, Tiirikainen M, Mani SA, Zhang W, Davuluri RV, Mimori K, Mori M, Sieuwerts AM, Martens JW, Tomlinson I, Negrini M, Berindan-Neagoe I, Foekens JA, Hamilton SR, Lanza G, Kopetz S, Fodde R, and Calin GA. 2013. CCAT2, a novel noncoding RNA mapping to $8 \mathrm{q} 24$, underlies metastatic progression and chromosomal instability in colon cancer. Genome Res 23:1446-1461. 10.1101/gr.152942.112

Ma G, Gu D, Lv C, Chu H, Xu Z, Tong N, Wang M, Tang C, Xu Y, and Zhang Z. 2015a. Genetic variant in 8q24 is associated with prognosis for gastric cancer in a Chinese population. Journal of Gastroenterology \& Hepatology 30:689-695.

Ma G, Gu D, Lv C, Chu H, Xu Z, Tong N, Wang M, Tang C, Xu Y, Zhang Z, Wang B, and Chen J. 2015b. Genetic variant in 8 q24 is associated with prognosis for gastric cancer in a Chinese population. J Gastroenterol Hepatol 30:689-695. 10.1111/jgh.12801

Marino JS, Stechschulte LA, Stec DE, Nestor-Kalinoski A, Coleman S, and Hinds TD, Jr. 2016. Glucocorticoid Receptor beta Induces Hepatic Steatosis by Augmenting Inflammation and Inhibition of the Peroxisome Proliferator-activated Receptor (PPAR) alpha. 291:25776-25788.

Mcbeth L, Nwaneri AC, Grabnar M, Demeter J, Nestor-Kalinoski A, and Hinds TD. 2016. Glucocorticoid receptor beta increases migration of human bladder cancer cells. Oncotarget 7:27313-27324.

Mercer TR, Dinger ME, and Mattick JS. 2009. Long non-coding RNAs: insights into functions. Nature Reviews Genetics 10:155-159.

Ozgur E, Ferhatoglu F, Sen F, Saip P, and Gezer U. 2019. Circulating IncRNA H19 may be a useful marker of response to neoadjuvant chemotherapy in breast cancer. Cancer Biomark. 10.3233/cbm-190085

Pan W, Liu L, Wei J, Ge Y, Zhang J, Chen H, Zhou L, Yuan Q, Zhou C, and Yang M. 2016. A functional IncRNA HOTAIR genetic variant contributes to gastric cancer susceptibility. Molecular Carcinogenesis 55:90-96.

Patki M, and McFall T. 2018. Chronic p27(Kip1) Induction by Dexamethasone Causes Senescence Phenotype and Permanent Cell Cycle Blockade in Lung Adenocarcinoma Cells Over-expressing Glucocorticoid Receptor. 
8:16006. 10.1038/s41598-018-34475-8

Prensner JR, Iyer MK, Balbin OA, Dhanasekaran SM, Cao Q, Brenner JC, Laxman B, Asangani IA, Grasso CS, and Kominsky HD. 2011. Transcriptome sequencing across a prostate cancer cohort identifies PCAT-1, an unannotated lincRNA implicated in disease progression. Nature Biotechnology 29:742-749.

Psarra AMG, Solakidi S, Trougakos IP, Margaritis LH, Spyrou G, and Sekeris CE. 2005. Glucocorticoid receptor isoforms in human hepatocarcinoma HepG2 and SaOS-2 osteosarcoma cells: Presence of glucocorticoid receptor alpha in mitochondria and of glucocorticoid receptor beta in nucleoli. Int J Biochem Cell Biol 37:2544-2558.

Qu F, Chen Y, Wang X, He X, Ren T, Huang Q, Zhang J, Liu X, Guo X, Gu J, and Xing J. 2015. Leukocyte mitochondrial DNA content: a novel biomarker associated with prognosis and therapeutic outcome in colorectal cancer. Carcinogenesis 36:543-552. 10.1093/carcin/bgv042

Ren Y, Shang J, Li J, Liu W, Zhang Z, Yuan J, and Yang M. 2017. The long noncoding RNA PCAT-1 links the microRNA miR-215 to oncogene CRKL-mediated signaling in hepatocellular carcinoma. Journal of Biological Chemistry 292:jbc.M116.773978.

Saeki N, Ono H, Sakamoto H, and Yoshida T. 2013. Genetic factors related to gastric cancer susceptibility identified using a genome-wide association study. Cancer Sci 104:1-8.

Sakamoto H, Yoshimura K, Saeki N, Katai H, Shimoda T, Matsuno Y, Saito D, Sugimura H, Tanioka F, and Kato S. 2008. Genetic variation in PSCA is associated with susceptibility to diffuse-type gastric cancer. Nature Genetics 40:730-740.

Sattarifard H, Hashemi M, Hassanzarei S, Narouie B, and Bahari G. 2017. Association between genetic polymorphisms of long non-coding RNA PRNCR1 and prostate cancer risk in a sample of the Iranian population. Mol Clin Oncol 7:1152-1158. 10.3892/mco.2017.1462

Shi WH, Wu QQ, Li SQ, Yang TX, Liu ZH, Tong YS, Tuo L, Wang S, and Cao XF. 2015. Upregulation of the long noncoding RNA PCAT-1 correlates with advanced clinical stage and poor prognosis in esophageal squamous carcinoma. Tumor Biology 36:2501-2507.

Shi Y, Hu Z, Wu C, Dai J, Li H, Dong J, Wang M, Miao X, Zhou Y, and Lu F. 2011. A genome-wide association study identifies new susceptibility loci for non-cardia gastric cancer at $3 q 13.31$ and 5p13.1. Nature Genetics 43:1215-1218.

Silvestri V, Rizzolo P, Scarno M, Chillemi G, Navazio AS, Valentini V, Zelli V, Zanna I, Saieva C, Masala G, Bianchi S, Manoukian S, Barile M, Pensotti V, Peterlongo P, Varesco L, Tommasi S, Russo A, Giannini G, Cortesi L, Viel A, Montagna M, Radice P, Palli D, and Ottini L. 2015. Novel and known genetic variants for male breast cancer risk at 8q24.21, 9p21.3, 11q13.3 and 14q24.1: results from a multicenter study in Italy. Eur J Cancer 51:2289-2295. 10.1016/j.ejca.2015.07.020

Song P, Jiang B, Liu Z, Ding J, Liu S, and Guan W. 2017. A three-IncRNA expression signature associated with the prognosis of gastric cancer patients. 6:1154-1164. 10.1002/cam4.1047

Sur I, Tuupanen S, Whitington T, Aaltonen LA, and Taipale J. 2013. Lessons from functional analysis of genomewide association studies. Cancer Research 73:4180-4184.

Tong Y, Wang H, Li S, Zhao F, Ying J, Qu Y, and Mu D. 2018. Cumulative evidence for relationships between multiple variants in 8q24 and colorectal cancer incidence. Medicine (Baltimore) 97:e11990. 10.1097/md.0000000000011990

Ulrich CM, Robien K, and McLeod HL. 2003. Cancer pharmacogenetics: polymorphisms, pathways and beyond. Nat 
494

495

496

497

498

499

500

501

502

503

504

505

506

507

508

509

510

511

512

513

514

515

516

517

518

519

520

521

522

523

524

525

526

527

528

529

530

531

532

533

534

Rev Cancer 3:912-920. 10.1038/nrc1233

Wadhwa R, Song S, Lee JS, Yao Y, Wei Q, and Ajani JA. 2013. Gastric cancer-molecular and clinical dimensions. Nature Reviews Clinical Oncology 10:643-655.

Wang W, Du M, Li Z, Zhang L, Li Q, Xu Z, Li B, Wang L, Li F, Zhang D, Xu H, Yang L, Gong W, Qiang F, Zhang Z, and Xu Z. 2018. A Genetic Variant Located in miR-146b Promoter Region Is Associated with Prognosis of Gastric Cancer. Cancer Epidemiol Biomarkers Prev 27:822-828. 10.1158/1055-9965.epi-17-1054

Wang X, Liu Y, Shao D, Qian Z, Dong Z, Sun Y, Xing X, Cheng X, Du H, Hu Y, Li Y, Li L, Dong B, Li Z, Wu A, Wu X, Bu Z, Zong X, Zhu G, Ji Q, Wen XZ, Zhang LH, and Ji JF. 2016. Recurrent amplification of MYC and TNFRSF11B in $8 q 24$ is associated with poor survival in patients with gastric cancer. Gastric Cancer 19:116-127. 10.1007/s10120-015-0467-2

Wang X, and Wang X. 2019. Long non-coding RNA colon cancer-associated transcript 2 may promote esophageal cancer growth and metastasis by regulating the Wnt signaling pathway. Oncol Lett 18:1745-1754. 10.3892/ol.2019.10488

Washington K. 2010. 7th Edition of the AJCC Cancer Staging Manual: Stomach. Annals of Surgical Oncology 17:3077-3079.

Weidle UH, Birzele F, Kollmorgen G, and Ruger R. 2017. Long Non-coding RNAs and their Role in Metastasis. Cancer Genomics Proteomics 14:143-160. 10.21873/cgp.20027

Wu ER, and Hsieh MJ. 2019. Association of IncRNA CCAT2 and CASC8 Gene Polymorphisms with Hepatocellular Carcinoma. 16. 10.3390/ijerph16162833

Xiang JF, Yin QF, Chen T, Zhang Y, Zhang XO, Wu Z, Zhang S, Wang HB, Ge J, and Lu X. 2014. Human colorectal cancer-specific CCAT1-L IncRNA regulates long-range chromatin interactions at the MYC locus. Cell Research 24:513-531.

Xiong Y, Wang R, Peng L, You W, Wei J, Zhang S, Wu X, Guo J, Xu J, and Lv Z. 2017. An integrated IncRNA, microRNA and mRNA signature to improve prognosis prediction of colorectal cancer. Oncotarget 8:85463-85478.

Y Ü, Dadaş E, Yalinbaş KB, Sümbül AT, Genç A, and Bayram S. 2017. The analysis of IncRNA HOTAIR rs12826786 C>T polymorphism and gastric cancer susceptibility in a Turkish population: lack of any association in a hospital-based case-control study. Irish Journal of Medical Science (1971 -) 186:859-865.

Yang ML, Huang Z, Wu LN, Wu R, Ding HX, and Wang BG. 2019. IncRNA-PCAT1 rs2632159 polymorphism could be a biomarker for colorectal cancer susceptibility. 39. 10.1042/bsr20190708

Yemelyanov A, ., Czwornog J, ., Chebotaev D, ., Karseladze A, ., Kulevitch E, ., Yang X, ., and Budunova I, . 2006. Tumor suppressor activity of glucocorticoid receptor in the prostate. Oncogene 26:1885.

Ying Y, Zhang X, Li Z, Deng L, Jiao G, Zhang B, Ping X, Mu H, Qiao W, and Jian Z. 2013. Glucocorticoid receptor $\beta$ regulates injury-mediated astrocyte activation and contributes to glioma pathogenesis via modulation of $\beta$-catenin/TCF transcriptional activity. Neurobiology of Disease 59:165-176.

Yuan Q, Chu H, Ge Y, Ma G, Du M, Wang M, Zhang Z, and Zhang W. 2018. LncRNA PCAT1 and its genetic variant rs1902432 are associated with prostate cancer risk. J Cancer 9:1414-1420.

Zeichner SB, Goldstein DA, Kohn C, and Flowers CR. 2017. Cost-effectiveness of precision medicine in gastrointestinal stromal tumor and gastric adenocarcinoma. Journal of Gastrointestinal Oncology 8:513523. $10.21037 /$ jgo.2016.04.03

Zhang $\mathrm{M}$, and Du X. 2016. Noncoding RNAs in gastric cancer: Research progress and prospects. World J Gastroenterol 22:6610-6618. 10.3748/wjg.v22.i29.6610

Peer) reviewing PDF | (2019:09:41373:1:0:REVIEW 3 Jan 2020) 
535

536

537

538

539

540

541

542

543

544

545

546

547

548
Zhao B, Hou X, and Zhan H. 2015a. Long non-coding RNA PCAT-1 over-expression promotes proliferation and metastasis in non-small cell lung cancer cells. Int J Clin Exp Med 8:18482-18487.

Zhao B, Hou X, and Zhan H. 2015b. Long non-coding RNA PCAT-1 over-expression promotes proliferation and metastasis in non-small cell lung cancer cells. International Journal of Clinical \& Experimental Medicine 8:18482.

Zhao J, Liu Y, Huang G, Cui P, Zhang W, and Zhang Y. 2015c. Long non-coding RNAs in gastric cancer: versatile mechanisms and potential for clinical translation. American Journal of Cancer Research 5:907.

Zheng Y, Izumi K, Li Y, Ishiguro H, and Miyamoto H. 2012. Contrary regulation of bladder cancer cell proliferation and invasion by dexamethasone-mediated glucocorticoid receptor signals. Molecular Cancer Therapeutics 11:2621.

Zhi P, Shi J, and Liu F. 2017. Genetic variations at $8 q 24$ and gastric cancer susceptibility: A meta-analysis study. PLoS One 12:e0188774. 
Table $\mathbf{1}$ (on next page)

Table 1 Characteristics of the GC patients 


\begin{tabular}{|c|c|c|c|}
\hline \multicolumn{2}{|c|}{ Variables } & \multirow{2}{*}{$\frac{\mathrm{N}}{340}$} & \multirow{2}{*}{$\begin{array}{r}\% \\
46.8 \\
\end{array}$} \\
\hline Age (years) & $<60$ & & \\
\hline & $\geq 60$ & 386 & 53.2 \\
\hline \multirow[t]{2}{*}{ Gender } & Male & 547 & 75.3 \\
\hline & Female & 179 & 24.7 \\
\hline \multirow[t]{2}{*}{ Smoking } & Yes & 281 & 39.0 \\
\hline & No & 440 & 61.0 \\
\hline \multirow[t]{2}{*}{ Drinking } & Yes & 198 & 27.4 \\
\hline & No & 524 & 72.6 \\
\hline \multirow[t]{2}{*}{ Family history } & Yes & 47 & 6.5 \\
\hline & No & 672 & 93.5 \\
\hline \multirow[t]{2}{*}{ H. pylori } & Positive & 438 & 68.7 \\
\hline & Negative & 200 & 31.3 \\
\hline \multirow[t]{2}{*}{ Tumour Size } & $<5 \mathrm{~cm}$ & 420 & 57.9 \\
\hline & $\geq 5 \mathrm{~cm}$ & 306 & 42.1 \\
\hline \multirow[t]{3}{*}{ TNM stage } & I & 129 & 17.8 \\
\hline & II & 271 & 37.3 \\
\hline & III & 326 & 44.9 \\
\hline \multirow[t]{3}{*}{ Histological type } & Tubular & 571 & 78.6 \\
\hline & Signet-ring cell & 68 & 9.4 \\
\hline & Other & 87 & 12.0 \\
\hline \multirow[t]{2}{*}{ Histological grade } & Low-grade & 218 & 30.0 \\
\hline & High-grade & 508 & 70.0 \\
\hline \multirow[t]{2}{*}{ Lymph vascular invasion } & Yes & 514 & 70.8 \\
\hline & No & 212 & 29.2 \\
\hline \multirow[t]{2}{*}{ Neural invasion } & Yes & 399 & 55.0 \\
\hline & No & 327 & 45.0 \\
\hline \multirow[t]{2}{*}{ Chemotherapy } & Yes & 314 & 43.3 \\
\hline & No & 412 & 56.7 \\
\hline
\end{tabular}

2 


\section{Table 2 (on next page)}

Table 2 Distributions of the genotypes of the gastric cancer patients

MST: median survival time, months. *Mean survival time was provided when MST could not be calculated 
Table 2 Distributions of the genotypes of the gastric cancer patients

\begin{tabular}{|c|c|c|c|c|c|c|c|}
\hline \multirow{2}{*}{$\frac{\text { Gene }}{\text { CCAT1 }}$} & \multicolumn{2}{|c|}{ Genotypes } & \multirow{2}{*}{$\frac{\text { Patients, } N}{495}$} & \multirow{2}{*}{$\frac{\text { Death, N (\%) }}{250(50.50)}$} & \multirow{2}{*}{$\frac{\text { MST }}{64.89}$} & \multirow{2}{*}{$\frac{\mathrm{HR}(95 \% \mathrm{CI})}{1.00}$} & \multirow{2}{*}{$\frac{\log \text { rank } P}{0.73}$} \\
\hline & rs 10087719 & $\mathrm{AA}$ & & & & & \\
\hline & & $\mathrm{AG}$ & 209 & $99(47.37)$ & 75.70 & $0.93(0.74,1.18)$ & \\
\hline & & GG & 20 & $8(40.00)$ & $54.67 *$ & $0.81(0.40-1.63)$ & \\
\hline \multirow[t]{3}{*}{ CCAT1 } & rs7816475 & GG & 572 & $280(48.95)$ & 70.04 & 1.00 & 0.65 \\
\hline & & $\mathrm{AG}$ & 134 & $69(51.49)$ & 56.38 & $1.08(0.83-1.41)$ & \\
\hline & & AA & 6 & $2(33.33)$ & $55.99 *$ & $0.61(0.15-2.45)$ & \\
\hline \multirow[t]{3}{*}{ PCAT1 } & rs1026411 & GG & 257 & $120(46.69)$ & 75.20 & 1.00 & 0.32 \\
\hline & & $\mathrm{AG}$ & 353 & $181(51.27)$ & 60.85 & $1.20(0.95,1.50)$ & \\
\hline & & AA & 111 & $53(47.75)$ & $63.07 *$ & $1.11(0.80-1.53)$ & \\
\hline \multirow[t]{3}{*}{ PRNCR1 } & rs 12682421 & AA & 443 & $230(51.92)$ & 58.51 & 1.00 & 0.03 \\
\hline & & GA & 248 & $106(42.74)$ & $68.84^{*}$ & $0.77(0.61-0.97)$ & \\
\hline & & GG & 33 & $21(63.64)$ & 37.78 & $1.25(0.80-1.95)$ & \\
\hline \multirow[t]{3}{*}{ PRNCR1 } & rs 13252298 & $\mathrm{AA}$ & 330 & $158(47.88)$ & 70.05 & 1.00 & 0.75 \\
\hline & & $\mathrm{AG}$ & 315 & $157(49.84)$ & 69.29 & $1.09(0.87-1.36)$ & \\
\hline & & GG & 79 & $42(53.16)$ & 55.39 & $1.07(0.76-1.51)$ & \\
\hline \multirow[t]{3}{*}{ CASC8 } & rs 1562430 & $\mathrm{TT}$ & 505 & $257(50.89)$ & 64.30 & 1.00 & 0.17 \\
\hline & & $\mathrm{CT}$ & 199 & $93(46.73)$ & 79.05 & $1.13(0.84-1.53)$ & \\
\hline & & $\mathrm{CC}$ & 19 & $6(31.58)$ & 75.20 & $0.68(0.35-1.16)$ & \\
\hline \multirow[t]{3}{*}{ CASC8 } & rs4871789 & GG & 251 & $129(51.39)$ & 64.89 & 1.00 & 0.64 \\
\hline & & $\mathrm{AG}$ & 344 & $165(47.97)$ & 73.50 & $0.90(0.71-1.13)$ & \\
\hline & & AA & 119 & $58(48.74)$ & 67.35 & $0.91(0.67-1.24)$ & \\
\hline \multirow[t]{3}{*}{ CASC8 } & rs6983267 & $\mathrm{TT}$ & 172 & $89(51.74)$ & 65.68 & 1.00 & 0.59 \\
\hline & & GT & 416 & $195(46.88)$ & 79.05 & $1.01(0.82-1.24)$ & \\
\hline & & GG & 95 & $48(50.53)$ & 67.35 & $0.93(0.84-1.09)$ & \\
\hline
\end{tabular}

2 MST: median survival time, months.

*Mean survival time was provided when MST could not be calculated 3 


\section{Table 3 (on next page)}

Table 3 Stepwise Cox regression analysis of gastric cancer survival

Age, gender, H. pylori, tumour size, TNM stage, histological type, histological grade, chemotherapy, lymph vascular invasion, neural invasion and eight SNPs polymorphism were used as variables in the regression model. 


\begin{tabular}{|c|c|c|}
\hline Genotypes & $P$ & Adjusted HR (95\% CI) \\
\hline Tumour size & 0.005 & $1.42(1.11-1.81)$ \\
\hline Lymph vascular invasion & $<0.001$ & $2.09(1.45-3.02)$ \\
\hline \multicolumn{3}{|l|}{ TNM stage } \\
\hline II vs I & 0.007 & $2.25(1.24-4.07)$ \\
\hline III vs I & $<0.001$ & $6.83(3.77-12.36)$ \\
\hline Chemotherapy & 0.007 & $0.72(0.56-0.91)$ \\
\hline rs12682421 (AA vs GA+GG) & 0.009 & $1.39(1.09-1.78)$ \\
\hline rs1562430 (TT vs CC+CT) & 0.016 & $1.38(1.06-1.80)$ \\
\hline rs1026411 (AA+AG vs GG) & 0.017 & $1.36(1.06-1.74)$ \\
\hline
\end{tabular}

2 Age, gender, H. pylori, tumour size, TNM stage, histological type, histological grade, chemotherapy, lymph vascular 3 invasion, neural invasion and eight SNPs polymorphism were used as variables in the regression model. 
Figure 1

Figure 1. Flow chart of the enrolled subjects

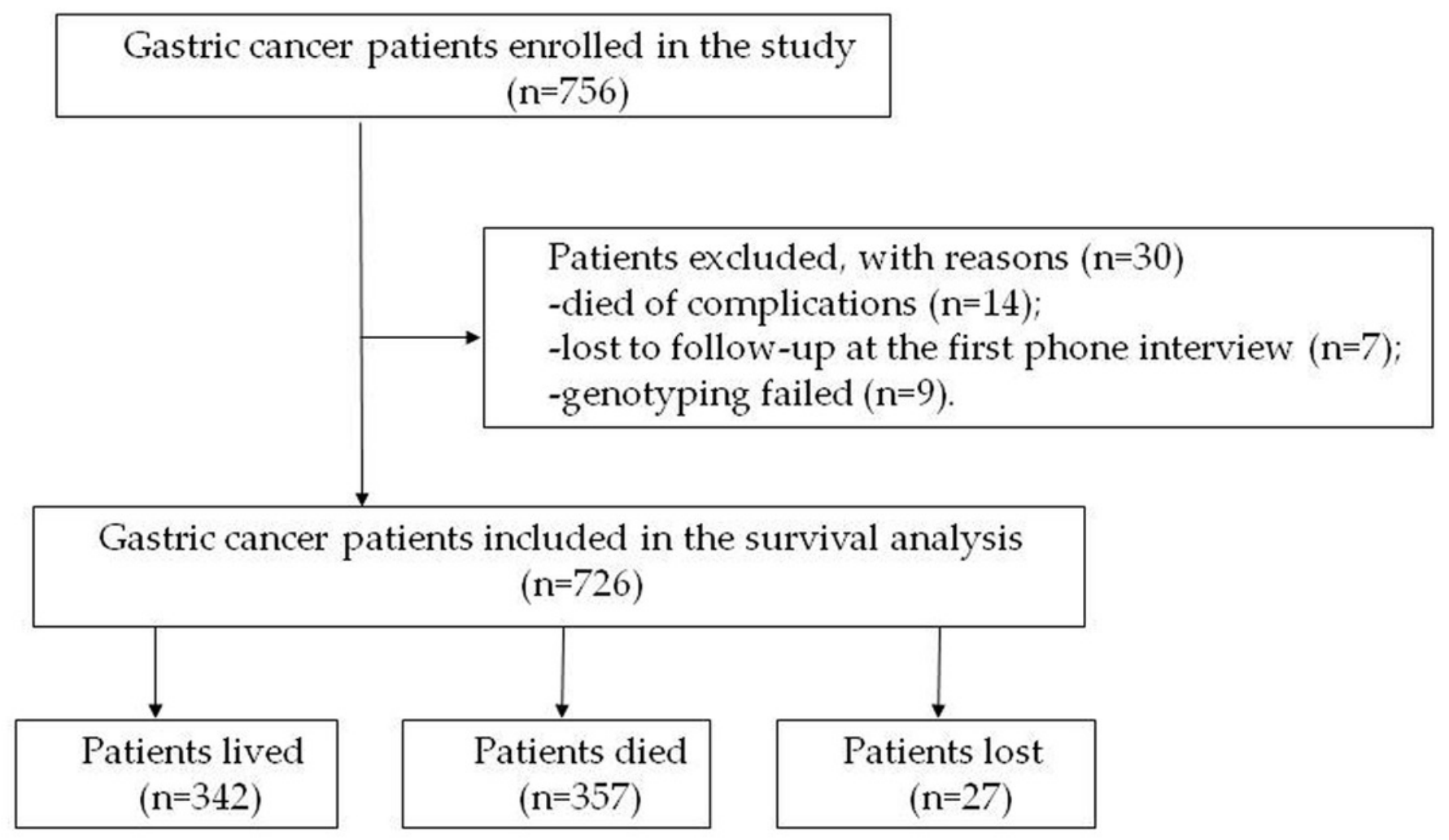


Figure 2

Figure 2. Association of genotypes with overall survival in gastric cancer patients.

A. Plot for rs12682421 using the dominant model (GG/GA vs. AA); B. Plot for rs1562430 using the dominant model (CC/CT vs. TT); C. Plot for rs1026411 using the dominant model (AA/AG vs.GG).
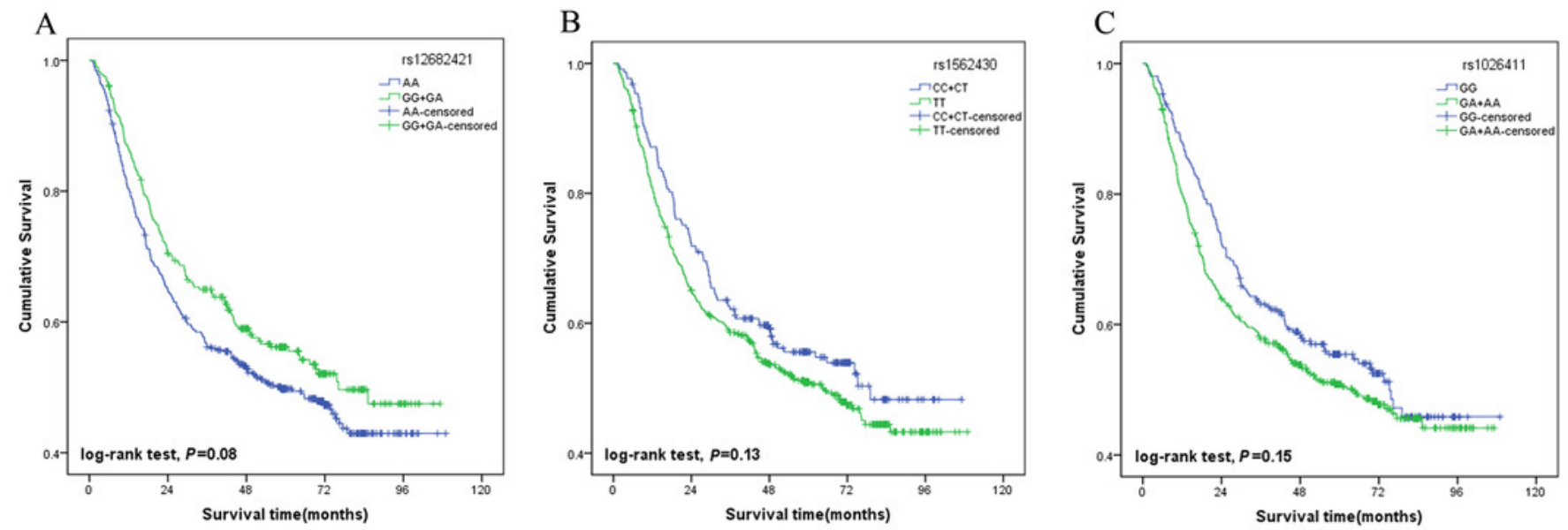


\section{Figure 3}

Stratified analysis for rs12682421, rs1562430 and rs1026411genotypes associated with gastric cancer patients' survival.

Age, gender, H. pylori, tumour size, TNM stage, histological type, histological grade, chemotherapy, lymph vascular invasion, neural invasion and eight SNP polymorphisms were used as variables in the regression model. A. Stratified analysis of rs12682421 genotypes associated with gastric cancer patients' survival (AA vs GA+GG); B. Stratified analysis of rs1562430 genotypes associated with gastric cancer patients' survival (TT vs CC+CT); C. Stratified analysis of rs1026411 genotypes associated with gastric cancer patients' survival (AA+AG vs $G G)$
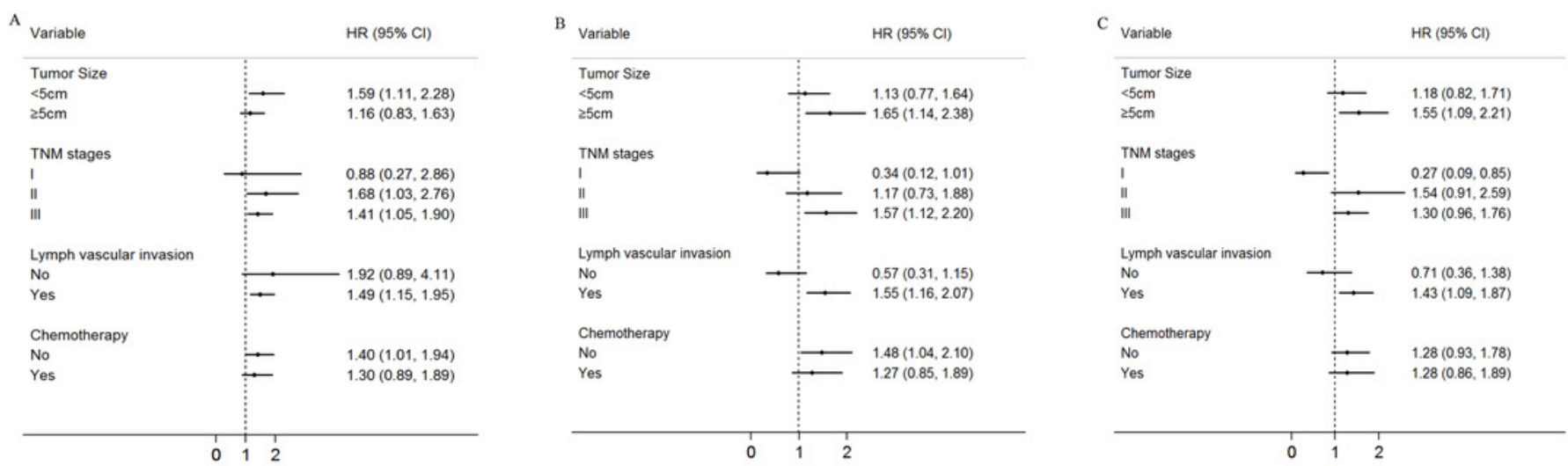